\title{
Altered Anatomical Modular Organization of Brain Networks in Patients with Major Depressive Disorder
}

\author{
Jing-Yu ZHU ${ }^{1}$, Xiang-Yu SHEN ${ }^{1}$, Jiao-Long $\mathrm{QIN}^{1}$, Mao-Bin WEI ${ }^{1}$, \\ Rui YAN ${ }^{2}$, Jian-Huai $\mathrm{CHEN}^{2}$, Zhi-Jian YAO ${ }^{2,3}$, Qing LU ${ }^{1,{ }^{*}}$ \\ ${ }^{1}$ Key Laboratory of Child Development and Learning Science (Ministry of Education), \\ Research Centre for Learning Science, Southeast University, Si Pailou 2, Nanjing, \\ 210096, China \\ ${ }^{2}$ Department of Psychiatry, Affiliated Nanjing Brain Hospital of Nanjing Medical \\ University, No. 264 Guangzhou Road, Nanjing, 210029, China \\ ${ }^{3}$ Nanjing Brain Hospital, Nanjing University Medical School, 22 Hankou Road, Nanjing, \\ 210093, China \\ ${ }^{*}$ Corresponding author
}

Keywords: Major depressive disorder, Modular organization, Structural connectivity.

\begin{abstract}
This study investigates the abnormal brain structural connectivity in patients with major depressive disorder (MDD) through a modularization approach. Diffusion tensor imaging (DTI) data were collected from 20 MDD patients and 20 age-matched controls. The structural modularity characteristics and their relationships with clinical variables were examined. Results indicated that MDD patients exhibited reorganized modules with widely damaged global inter-module connectivity in limbic system and altered intra-module connectivity in left hemisphere. Furthermore, the module degree in right rolandic operculum, left posterior cingulate gyrus, right cuneus and right fusiform gyrus were negatively correlated with the depression severity. Additionally, a positive relationship was also found between the duration of disease and participant coefficient in left median cingulate. The current findings highlight altered modular organization in MDD patients, which might contribute to the pathogenesis of the disease.
\end{abstract}

\section{Introduction}

Major depression was the third leading cause of burden among all diseases in the year 2004 and it is expected to be the greatest cause in 2030 [1]. Depressed patients had been shown widely distributed functional and structural abnormalities in neuroimaging studies [2, 3].

Modularity is a novel quantitative measures of topological properties in the global brain level [4], and were used widely in studies of brain networks [5, 6]. Peng et al [4] found altered patterns of brain module network at the global level in MDD patients. Nevertheless, it remains unknown that whether the modular organization of anatomical brain networks is abnormal in patients with MDD.

In this paper, diffusion tensor imaging (DTI) was used to investigate the topological infrastructure of brain anatomical networks in MDD patients. Many studies applied DTI to detect MDD-related alterations in white matter [7] and primarily concentrated on fractional anisotropy metric. The current study tests two hypotheses: (1) the structural modularity in brain network would be disrupted in MDD patients; (2) the topological pattern alterations may be related with clinical factors of MDD. 


\section{Materials and Methods}

\section{Subjects}

Twenty patients with MDD were recruited from the Department of Psychiatry, Nanjing Brain Hospital, China. Depression severity was rated using a 17-item Hamilton Rating Scale for Depression (HAM-D). Twenty comparison healthy subjects with matched gender-, age-, handedness- were recruited via advertisements (shown in Table 1).

Table 1. Demographic and Clinical Characteristics of the Subjects

\begin{tabular}{|l|l|l|l|}
\hline Variables & HC $(\mathrm{n}=20)$ & DP Baseline $(\mathrm{n}=20)$ & $p$ value \\
\hline Age(years) & $23-54(40.70 \pm 10.52$ & $23-53(40.14 \pm 9.34)$ & 0.256 \\
\hline Educational level(years) & $5-18(11.60 \pm 4.11)$ & $5-15(10.80 \pm 2.95)$ & 0.078 \\
\hline Gender(male/female) & $11 / 9$ & $11 / 9$ & 1 \\
\hline Handedness(Right/Left) & $20 / 0$ & $20 / 0$ & 1 \\
\hline Score of 17-item HAMD & - & $42.89 \pm 7.30$ & - \\
\hline Duration of illness (months) & - & $5.08 \pm 7.41$ & - \\
\hline
\end{tabular}

\section{Scan Acquisition}

Magnetic resonance imaging utilized a 1.5 Tesla GE Signa scanner. T1-weighted axial images parameters included: repetition time $=500 \mathrm{~ms}$; echo time $=14 \mathrm{~ms}$; thickness $=1.0 \mathrm{~mm}$; gap $=0 \mathrm{~mm}$; flip angle $=15^{\circ}$. Parameters of DTI images included: repetition time $=10,000 \mathrm{~ms}$; echo time $=81.2 \mathrm{~ms}$; thickness $=4.0 \mathrm{~mm}$; gap $=0 \mathrm{~mm}$.

\section{Data Preprocessing}

Data preprocessing was carried out using the Diffusion Toolbox of FMRIB's software library (http://www.fmrib.ox.ac.uk/fsl/fdt/index.html). The following steps were performed: eddy current and motion artifact correction of the raw data; estimation of the diffusion tensor; computation of the fractional anisotropy (FA).

\section{Structural Network Construction}

The brain template of the automated anatomical labeling atlas (AAL) [8] was adopted to define the nodes of networks, with 45 regions of interest for each hemisphere [9]. White matter tract reconstruction was performed based on the Fiber Assignment by Continuous Tracking (FACT) algorithm [10] (http://www.trackvis.org). The connection of two nodes was determined by a fiber that touched both nodes $i$ and $j$, while the weights of edges were defined as mean FA values of the streamlines.

\section{Modular Organizations}

Group Network. Modular organizations were defined based on the group-averaged network. In this study, only connections that were present in at least $80 \%$ of population of a group were retained to avoid fictitious joinings caused by noise, while all other connections were set to 0 .

Network Modularity. Module is defined as a subset of nodes tightly connected within the modules but sparsely connected between the modules. The modularity in the paper was computed using the spectral algorithm [11]. For global level, $\mathrm{IMC}_{i, j}$ was defined as the inter-module connectivity between module $i$ and module $j$, while $\mathrm{MC}_{i}$ was described as the intra-module connectivity within module $i$ [4]. For regional level, 
the participation coefficient (PC) and module degree (MD) were to measure the regional role played by each brain region [12].

\section{Statistical Analysis}

To evaluate the statistical significance of modularity differences, a non-parametric permutation testing was implemented [5], and a false discovery rate (FDR) correction was performed with the threshold of $p=0.05$. The correlations between clinical scores and the modular features in patients group were explored by means of a Pearson correlation method, and the outliers were identified according to the strategy proposed by means of the work of Schwarzkopf et al [13].

\section{Results}

\section{Modular Organizations}

We detected five modules in the healthy controls (Fig. 1A) and four modules in the MDD patients (Fig. 1B). Brain modes in the two groups were visualized with the BrainNet Viewer (http://www.nitrc.org/projects/bnv/) [14].

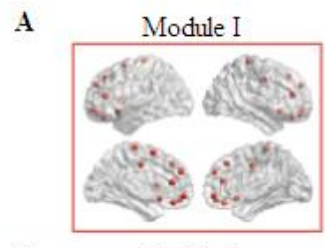

B

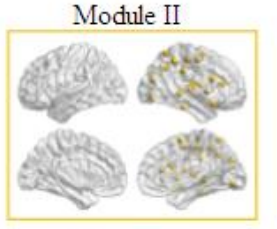

Module II

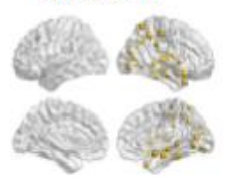

Module III

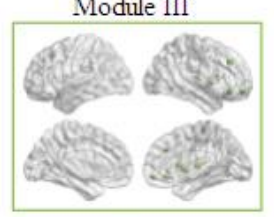

Module III

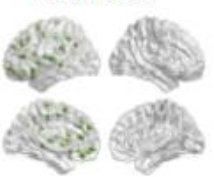

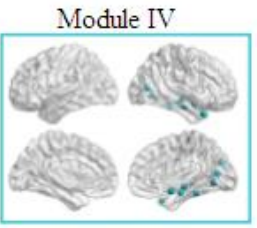

Module IV

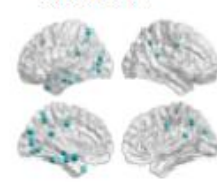

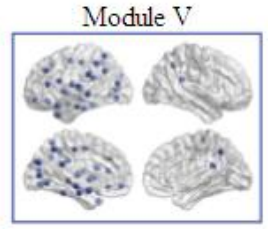

Figure 1. Modular organization of the brain structural network.

\section{Brain Modularity}

Global Level Modular Characteristics. Global brain modularity in module IV and $\mathrm{V}$ of MDD patients changed a lot when comparing with healthy controls (see Table 1).

Table 2. Inter-/Intra- module connections in MDD group compared with healthy group

\begin{tabular}{|l|l|}
\hline Global level modular characteristics & $p$ value \\
\hline $\mathrm{IMC}_{14}$ & $0.0124 \downarrow$ \\
\hline $\mathrm{IMC}_{24}$ & $0.0092 \downarrow$ \\
\hline $\mathrm{IMC}_{34}$ & $0.0013 \downarrow$ \\
\hline $\mathrm{IMC}_{35}$ & $0.0482 \downarrow$ \\
\hline $\mathrm{MC}_{4}$ & $0.0362 \downarrow$ \\
\hline $\mathrm{MC}_{5}$ & $0.0099 \uparrow$ \\
\hline
\end{tabular}

$\uparrow$ indicated that the value was increased in MDD group.

$\downarrow$ indicated that the value was decreased in MDD group.

Regional Level Modular Characteristics. Compared to the healthy controls, most regions in each modules in patients group showed significant alterations (Fig. 2). 


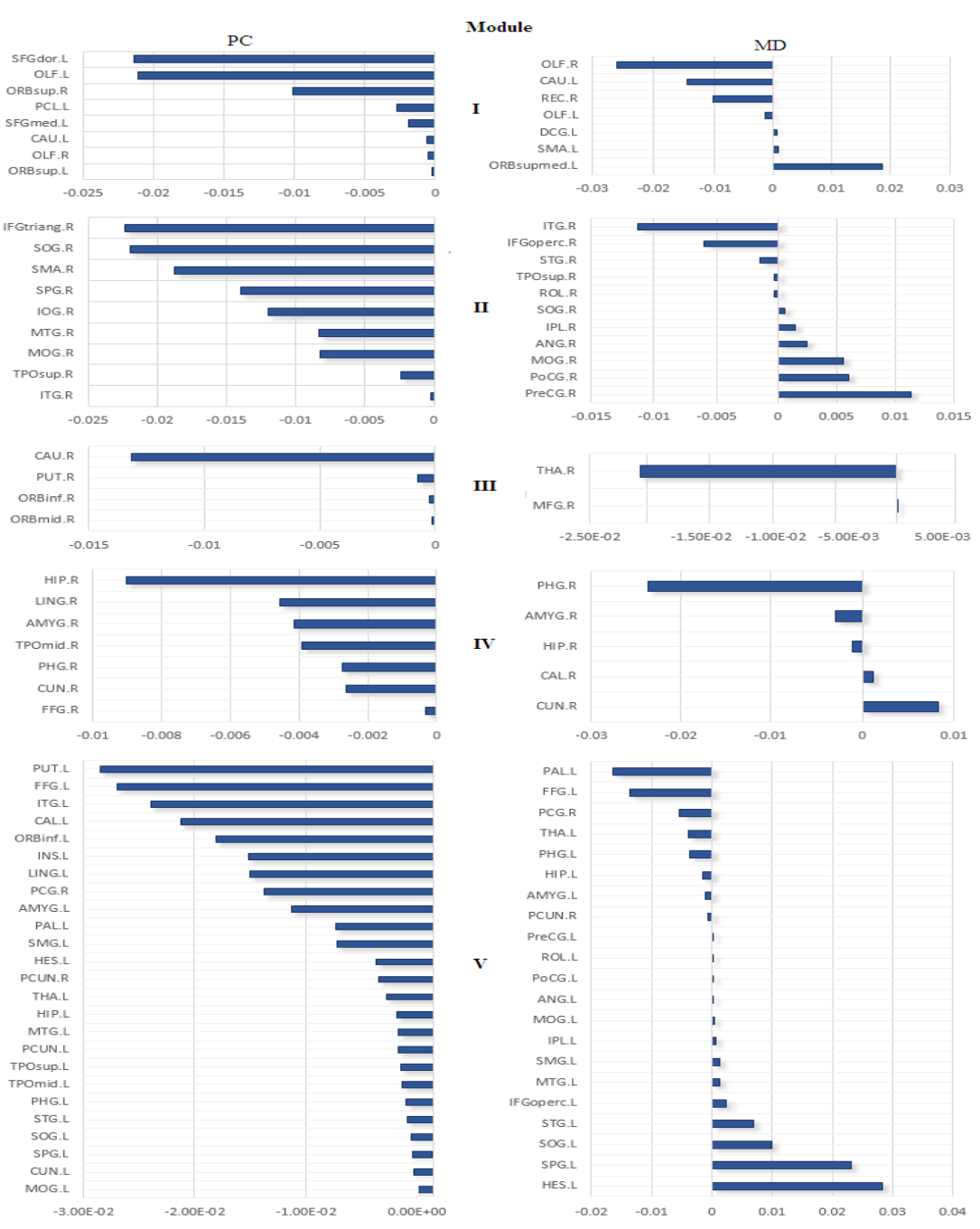

Figure 2. Between group differences of PC and MD in module network

\section{Relationships between Modularity and Clinical Variables}

The results showed that MD in the right Rolandic operculum (Module II) had a negative relationship with the 17 items' HAMD, and MD in the left posterior cingulum (Module V) was negatively correlated with the HAMD score. In addition, both right cuneus and right fusiform in Module IV showed positive related MD value with 17 items' HAMD. PC in the left middle cingulum in Module I showed significant positive correlation with the duration of the disease (Fig. 3).
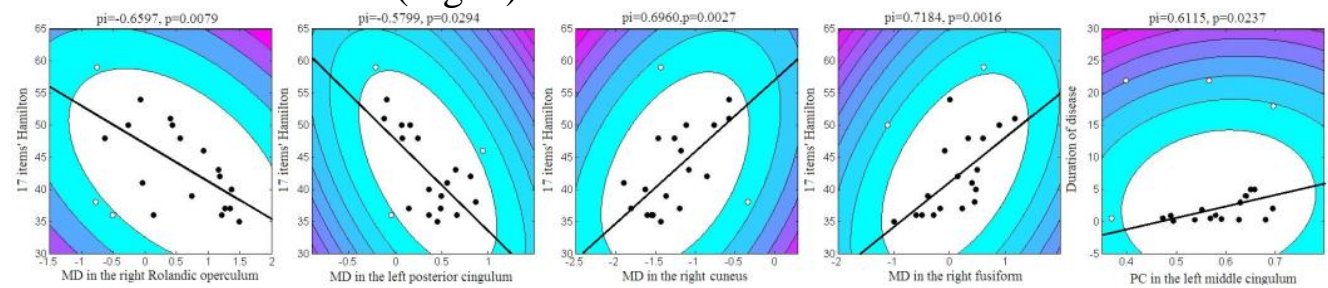

Figure 3. Relationships between regional level modularity characteristics and clinical scores

\section{Discussion}

To the best of our knowledge, the current study is the first to investigate the modularity of structural brain network and its relationship with clinical severity and duration of the disease in MDD patients. Results indicated that among patients group, the symmetry 
between two hemispheres in Module I was damaged. Module II, III and IV were rearranged while module $\mathrm{V}$ was divided into two parts.

In assessing the global level module connectivity, significant diminished IMC values were found in MDD patients. Moreover, the intra-module connectivity MC within Module IV showed significant decrease in patients group, while in Module V was higher than healthy controls. Both IMC and MC in patients group got deterioration in Module IV, which was consisted of right hippocampus, parahippocampus, amygdala, calcarine, cuneus, lingual and fusiform. These areas belong to the limbic system and is responsible for the consolidation of information from short-term memory to long-term memory and spatial navigation[15], suggesting that these regions and their neighbors were integrated, which is in accordance with previous studies.

Our study found the general decreased PC in MDD suggesting disrupted inter connectivity among each modules(Fig. 2). Meanwhile, MDD patients got increased MD mainly in the frontal, occipital, parietal areas and decreased MD in the prefrontal, subcortical and temporal areas.

Another finding of this study was that the relevance between regional modularity characteristics and clinical severity: MD in four regions (right Rolandic operculum, left posterior cingulum, right cuneus, right fusiform) showed significant relationship with 17 items' HAMD scores. And PC in the left middle cingulate was positively related with the duration of the disease. The Rolandic operculum belongs to auditory network, and had also been detected as a feature with discriminative power when classifying MDD patient from a healthy person[16].

Furthermore, left posterior cingulum may be a potential biomarker for severity of Major depressive disorder. And abnormalities of right cuneus and fusiform in visual recognition network were also found to be related with clinical variables. Previous study suggested that the damaged function of fusiform gyrus and prefrontal cortex in memory processing might contribute to cognitive vulnerability in MDD patients[17].

\section{Acknowledgement}

The work was supported by the grants of: the National High-tech Research and Development Program of China (2015AA020509); the National Natural Science Foundation of China (81571639, 81371522, and 61372032); the Clinical Medicine Technology Foundation of Jiangsu Province (BL2014009); the Natural Science Foundation of Jiangsu Province (BK20131074).

\section{References}

[1] A. Cipriani, M. Purgato, T. A. Furukawa, C. Trespidi, G. Imperadore, A. Signoretti, et al., "Citalopram versus other anti-depressive agents for depression," Cochrane Database Syst Rev, vol. 7, p. CD006534, 2012.

[2] L. L. Zeng, H. Shen, L. Liu, L. Wang, B. Li, P. Fang, et al., "Identifying major depression using whole-brain functional connectivity: a multivariate pattern analysis," Brain, vol. 135, pp. 1498-507, May 2012.

[3] C. Diener, C. Kuehner, W. Brusniak, B. Ubl, M. Wessa, and H. Flor, "A meta-analysis of neurofunctional imaging studies of emotion and cognition in major depression," Neuroimage, vol. 61, pp. 677-85, Jul 22012. 
[4] D. Peng, F. Shi, T. Shen, Z. Peng, C. Zhang, X. Liu, et al., "Altered brain network modules induce helplessness in major depressive disorder," J Affect Disord, vol. 168, pp. 21-9, Oct 2014.

[5] K. Wu, Y. Taki, K. Sato, S. Kinomura, R. Goto, K. Okada, et al., "Age-related changes in topological organization of structural brain networks in healthy individuals," Hum Brain Mapp, vol. 33, pp. 552-68, Mar 2012.

[6] Y. Sun, Q. Yin, R. Fang, X. Yan, Y. Wang, A. Bezerianos, et al., "Disrupted functional brain connectivity and its association to structural connectivity in amnestic mild cognitive impairment and Alzheimer's disease," PLoS One, vol. 9, p. e96505, 2014.

[7] C. E. Sexton, C. E. Mackay, and K. P. Ebmeier, "A systematic review of diffusion tensor imaging studies in affective disorders," Biol Psychiatry, vol. 66, pp. 814-23, Nov 12009.

[8] N. Tzourio-Mazoyer, B. Landeau, D. Papathanassiou, F. Crivello, O. Etard, N. Delcroix, et al., "Automated anatomical labeling of activations in SPM using a macroscopic anatomical parcellation of the MNI MRI single-subject brain," Neuroimage, vol. 15, pp. 273-89, Jan 2002.

[9] A. Zalesky, A. Fornito, I. H. Harding, L. Cocchi, M. Yücel, C. Pantelis, et al., "Whole-brain anatomical networks: Does the choice of nodes matter?," NeuroImage, vol. 50, pp. 970-983, 2010.

[10]S. Mori, B. J. Crain, V. P. Chacko, and P. C. M. Van Zijl, "Three-dimensional tracking of axonal projections in the brain by magnetic resonance imaging."

[11]M. E. Newman, "Modularity and community structure in networks," Proc Natl Acad Sci U S A, vol. 103, pp. 8577-82, Jun 62006.

[12]R. G. a. L. A. N. Amaral, "Functional cartography of complex metabolic networks," NIH Public Access, vol. 433(7028), pp. 895-900, 2005.

[13]D. S. Schwarzkopf, B. De Haas, and G. Rees, "Better ways to improve standards in brain-behavior correlation analysis," Front Hum Neurosci, vol. 6, p. 200, 2012.

[14]M. Xia, J. Wang, and Y. He, "BrainNet Viewer: A Network Visualization Tool for Human Brain Connectomics," PLoS ONE, vol. 8, p. e68910, 07/0404/19/received06 /08/accepted 2013.

[15]H. Eichenbaum, "A cortical-hippocampal system for declarative memory," Nat Rev Neurosci, vol. 1, pp. 41-50, 10//print 2000.

[16] J. Qin, M. Wei, H. Liu, J. Chen, R. Yan, Z. Yao, et al., "Altered anatomical patterns of depression in relation to antidepressant treatment: Evidence from a pattern recognition analysis on the topological organization of brain networks," J Affect Disord, vol. 180, pp. 129-37, Jul 152015.

[17] G. A. van Wingen, P. van Eijndhoven, H. R. Cremers, I. Tendolkar, R. J. Verkes, J. K. Buitelaar, et al., "Neural state and trait bases of mood-incongruent memory formation and retrieval in first-episode major depression," J Psychiatr Res, vol. 44, pp. 527-34, Jun 2010. 